Expert systems can increase the effectiveness of planning. Planning information can be exchanged through intelligent computer/communication aids. More effective knowledgebased management of planning projects is possible through the use of such aids in distributed networks. We discuss the potential and limitations of such networks.

\title{
Distributed Expert Systems for Planners
}

\author{
MANFRED KOCHEN \\ CHARLES BARR \\ University of Michigan
}

When the Aswan Dam was planned, there was recorded as well as tacit knowledge about its unwanted side effects, such as the spread of schistosomiasis and diminished soil fertility. The fact that Egyptian experts now regard its benefits to greatly outweigh the costs and disutilities does not excuse lack of better planning. The knowledge may not have been retrieved and brought to bear at the right time. If it was, it may not have played an appropriate role in the planning process.

We have yet to learn how to fully utilize relevant knowledge in planning. We need first to understand what knowledge it takes to plan various courses of actions that pursue a given policy. We need to determine whether that needed knowledge exists. If we believe it to exist, we need to learn how to retrieve it, possibly transform it so we can use it, screen and evaluate it, and bring it to bear at the right time. We must learn how to inject it into the planning process as high-technology, knowledge-based expert systems become available to planners.

In the next section, we ask if the effective utilization of knowledge in planning is too low and, if so, why. What would its more effective use mean? Our main goal in the third section is to explore the potential of distributed intelligence in networks that link machines, people, and problem-solving actions. We ask what kind of intelligent knowledge- 
networking system could be provided to increase the effectiveness of planning. Multiactor planning demands a blend of consensus formation and negotiation, for which the complexity of organization of information will be much greater than for a plan executed by a single responsible "planner." In the latter case, a logical specification of steps and contingencies will suffice for the goals selected by the planner. For large systems of actors and consequences, goals and means will be far more changeable. Communication will be needed if more effective planning is to be done. In the final section we discuss some of the problems involved in bringing such networks about, and conclude with recommendations for realistic action.

\section{Effectiveness of Knowledge-Use in Planning}

To plan is to specify spatial and temporal correspondences of actions believed to lead to a desired future state. Many stakeholders, professionals, politicians, and others are involved in planning any complex activity. A major source of complexity in planning, which creates barriers to effective knowledge utilization, is that no two planning participants interpret the key concepts in the same way.

Desired future states are popularly called ends, and actions intended to attain them are called means. Feldt (1986) has proposed classifying planning situations according to the extent to which (1) the ends are clearly known, and (2) the means are clearly known. Dichotomizing these two degrees of clarity, he considers four types of planning situations for which four types of "theory" (actually belief systems or "isms") are appropriate: rationalism, when both means and ends are clearly known; incrementalism, when neither means nor ends are clearly known; utopianism, when ends are clearly known but means are not; and methodism, when means are clearly known but ends are not. "Clarity of knowledge" is, however, subject to a great deal of variability in interpretation. The concepts of structured, semistructured, and unstructured have been used by Simon. It is probably safe to assume that most writers would agree that the precise specification of relatively few variables to characterize a state or an action corresponds to clear knowledge, whereas vaguely felt but unarticulated states and actions correspond to unclear knowledge. In between lie descriptions involving many interrelated, poorly specified variables close to the unclear pole 
and variables specified probabilistically or by fuzzy theoretic grades of membership closer to the clear pole.

We cannot expect the same degree of effectiveness in knowledge use for different planning situations. Situations in which rationalism is appropriate call for a high degree of knowledge utilization. But incrementalism, whether it employs reflection-in-action (Schon, 1984) or a "learning" planning style (Friedman and Abonyi, 1976; Michael, 1973), also requires knowledge. The former uses deep knowledge recorded in the literature and is in the form of general principles and long chains of reasoning; the latter relies on experience and relatively broad and shallow knowledge in the form of heuristics for how to do something in a specialized domain. Idealized states and actions in the rationalistic approach correspond to those studied by a physicist when, for example, he or she seeks to accelerate a particle to a high-energy state by applying a sequence of small forces to it, as it accelerates, much as a parent adds small pushes to a child on a swing. The set of all possible states, as well as of all possible actions that can be taken in each and the resulting change of state, is what is to be clearly known and specified as a first step. The value of each state or of regions in state-space to the planners, or at least some preference ordering over the states, must be clearly specified and known as a second step. There must also be clear knowledge of the present state from which planning is to proceed. The same holds for the desired future state.

Lest it be thought that incrementalism fits the above model with the desired future state reachable from the starting state in one incremental step, that is not the intent of writers about this approach. Neither the present state nor any future state is clearly specified; it is felt to be preferable to the starting state after it is encountered, but heuristics sometimes guide the planner about which action to choose. Thus, there are experts in "muddling through," and it is their expertise that constitutes knowledge. Given the limitations to how much planning effectiveness can be expected with this approach, this kind of knowledge has probably been used effectively.

Effective planning requires more interpretive aids to guide the use of various kinds of knowledge for these various planning environments. It requires sophisticated aids for discriminating among types of knowledge. Knowledge is needed by those playing roles as communicators, interpreters, and decision makers. Value-laden choices are evident at all levels of planning: For example, at the level of small projects, controversy arises in neighborhoods and cities over the siting of halfway 
houses for ex-convicts or mental patients. But the information needed for planning large complex projects, and evaluating their effects in social, economic, and environmental systems, is greater than for small ones. Large projects are a challenge to information management as well as knowledge interpretation. Knowledge-transfer innovation is needed because of the diversity and complexity of the institutions participating in such projects.

Low effectiveness of policymaking and planning is also partly a function of a project or planning timeframe being greater than the "visible horizon" or "planning foresight." Oversimplification of complex issues may also contribute to low effectiveness, but knowledge management may counter the negative aspects of such reductionism. Uncertainties and changes in the "out years" undermine the long-term effectiveness of decisions made now. Second, parties to planning have unequal time horizons, unequal needs to economize, and unequal pressures to represent constituencies rigidly. Third, any decision, plan, or policy addresses an artificially constructed problem. This may be viewed as a closed system. It may also be seen as a system with oversimplified cause and effect and feedback loops to the rest of the environment. A solution to these problems is designing learning systems that detect and correct error and thus support adaptive planning (Michael, 1973). This avoids a combinatorial explosion of forecasting all possible world states. Still, investments of real, limited resources must be made in the present. Feedback should be available within a planner's timeframe to help inform choices of resource investments. Measures of effectiveness chosen to meet these general needs must come to grips with the complexity of decision making in different styles of planning.

\section{Increasing Effectiveness Through Use of Distributed Expert Systems}

Our proposal for an interactive distributed expert-system network aims at raising the effectiveness of planning by extending mind power, bridging conceptual gaps, exchanging insights among planning stakeholders, integrating diverse knowledge, and facilitating satisfactory consensus formation and negotiation. Conveying research as well as routine knowledge through planning information systems would be one 
activity such networks could support. The kind of system we propose consists of

(1) people among whom consensus is required for a policy and a plan to work, and who are to be supported by the remaining five components;

(2) procedures for their use of the subsystems;

(3) models for their use, particularly inference engines (deductive and inductive);

(4) data bases, knowledge bases, and bibliographic data bases combined into expert systems connected to the inference engines;

(5) software, such as on-line advisory expert systems used as consultants, automated tutorial assistants, and utility programs that make the system so easy to use that it does not divert novice or casual users from their main task; and

(6) a distributed computer communication system with store-and-forward facilities, conferencing, and other capabilities needed to support 1-5. Figure 1 sketches the overall structure of the system.

Only a fraction of all the actors involved have sufficient power and influence to shape the policies and plans at the highest level. This group also motivates others to participate, and it sanctions the debate. Identifying these people is a critical success factor in planning. Any one of them can veto or sabotage a proposed policy or plan, and the agreement of each one is essential to success. Each one of them is therefore assumed to be a participant in the system, with ready access to it. Each owns as many as three terminals or personal computers usable as terminals: one in the office, if appropriate; a portable one for use in the field, car, farm, or site; one at home, where appropriate.

Suppose that a motion for a particular proposal has been put before this group by one of its members, for resolution within a few weeks: for example, to assess the impacts of a plan and analyze its uncertainties, risks, and potential environmental and economic effects. The choice of agenda and time limit is flexible. If adding another month is felt to change the outcome, it may be done. If they use their terminals, they will be notified of this action item; but even if they don't sign on or turn on their terminal, it will ring and blink at them until they do. (The MacIntosh, for example, has a continually active clock.) The proposal presents as objective and well-researched a case, taking into account the benefits and costs to all concerned, as the proposer can make without disguising his or her self-interest, if any. At first, everyone is asked to detect and reduce possible errors of commission and omission or of 


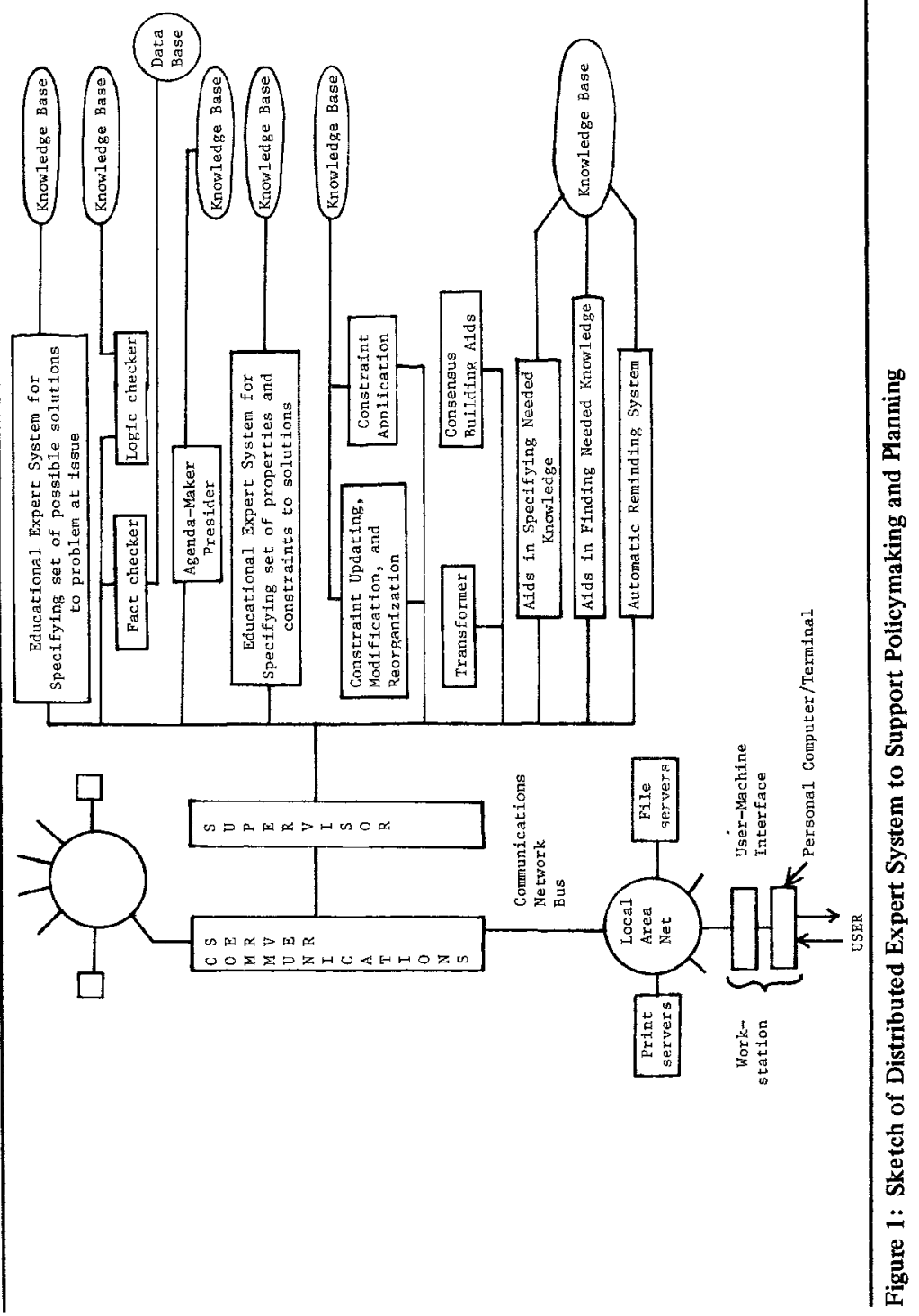


stating the wrong problem or representing it improperly. They should check if all the key decision makers are participating. A major objective at this stage is to look at a set of possible solutions in the formulation of the problem that is qualitatively and quantitatively appropriate-not too many, not too few, not too rich and sophisticated, not too poor. This requires consensus building by synthesis of diverse worldviews or representations of the situation and effecting some shifts of representation and some compromises. An educational subsystem embodying expertise in "getting to yes" (Fisher and Ury, 1981) about representations could help do this. Games involving role playing and serving to improve communication-somewhat like corporate group therapyhave proved to be valuable tools in effecting such changes (Duke, 1984). Of course, generating a custom-made game in a few weeks is unlikely unless new computer aids to game construction are developed.

The system is to help the participants detect differences in their representations, to diagnose from their expressions how they differ or concur in their understanding of the issues. Each participant may have a personalized expert system to better help him or her understand the issue, but it is connected to a systemwide expert system in somewhat the way the various views of a data base relate to the data base administrator's view (i.e., to the conceptual schema). The overall system is to suggest ways of reducing differences.

A simpler function is to check factual and logical inputs. Participants may challenge an input and request the checker to act. But the checker can be turned on at all times. Both deductive and inductive (statistical) inferences are checked, with results played back to all participants and used to update the record. The checking program is a consensually approved module, based on its performance on test cases.

The next phase of system use, guided by a human discussion leader or, optionally, by turning on an automatic agenda maker/presider, is consensus building of properties and constraints a solution to the problems at issue should satisfy. This requires shifts and compromises among values and interests after differences and commonalities have been made explicit. This is to be aided by an educational expert (sub) system that embodies "getting to yes" about values. This is similar in structure to the system for facilitating consensus building about representations. This is far less knowledge and logic-intensive and much more politically, psychologically, and socially sensitive, but at least one experiment has demonstrated the promise of computer conferencing for shifts in value (Kochen and Crickman, 1978). 
Constraints are constantly added and modified during both policymaking and planning. Deadlines and strategic timing-and elastic constraints about these-are important factors. So are flexible budgets and resource constraints, including capacities for computation and communication. Cultural, psychological, social, and political constraints are at least as important. All these must be stored in a continually growing and reorganized knowledge base, interfaced by a system that brings these constraints to bear at the right time.

Recent publications explore the idea of the need for better systematization of information about project environmental effects. Dissemination of expertise in problem solving and planning processes is perceived as an effectiveness-increasing management tool. The National Academy of Science recommends that large-scale, knowledge-growth experiments be integrated opportunistically into environmental impact analysis efforts (National Research Council, 1986).

The next phase in system use, probably overlapping with unterminated earlier phases, is the search for and use of knowledge that is necessary and sufficient to find a solution within the set of possible solutions and satisfying the agreed-upon constraints. We need to consider seven possibilities.

- First, the needed knowledge may be readily available, but the participants cannot integrate as well the noncognitive factors that play a role. It may seem to diminish what a participant thinks he or she knows.

- In the second case, useful knowledge exists, but no one thinks of its existence or of using it at the time it would help. An automatic reminder aid could help in this regard (Kochen and Floyd, 1983).

- In the third case, useful knowledge exists, is thought of, but cannot be retrieved in time or in a form to be used. An improved information document retrieval system can help here.

- In the fourth case, potentially useful knowledge has been retrieved. But because it may have been generated for a different purpose and require transformation, a system to assist in such a transformation may be needed. It may be automatically called on or, if special difficulties in transformation arise, a subsystem offers advice to this effect.

- Fifth, potentially useful knowledge may exist, but no one can specify what it is or recognize it.

- Sixth, the needed knowledge does not yet exist. That, presumably, is believed to be the case for the water project for which a study of alternatives has been launched.

- Seventh, we cannot know all we need to know to formulate perfectly effective policies and plans. That might hold for most cases. System use at 
any time is characterized at best by a probability distribution over these seven possibilities and perhaps quite often by confusion.

The planning abilities and actions supported by the network system described in this section are likely to increase in effectiveness for the following reasons. First, the system components provide for much better acquisition and interpretation of knowledge, which are needed for meeting the different kinds of planning challenges described earlier. Many kinds of planning have an arational character. Second, we propose to decentralize and ease access to the technology for all planning participants, thus seeking to assure that the knowledge and knowledgeprocessing resources of planning are not in scarce supply for crucial planning participants. Third, the system design is intended to carry out a search for knowledge, of the type and at the times needed, more effectively than humans acting alone. We need now to address the feasibility of such a system.

\section{Feasibility Issues}

Assessing feasibility of the system requires appreciating the difficulty of problems yet to be solved and understanding some basic issues. The distinction between data, information, knowledge, understanding, and wisdom is relevant (Kochen, 1975). This article has so far blurred it by calling all these information.

Observing or using an instrument generates data. If the data can be interpreted in an uncertainty reducing context, in which the scales of the instrument are explicitly presented with the data, the result is information. Of particular relevance to planning are three kinds of uncertainty: factual (technical or social); value-laden, based on opinions and reflecting priorities; and relating to actions by other affected organizations in the same area.

Much as the units of knowledge in a knowledge system are clusters of information items imbued with meaning, the units of understanding are clusters of meta-information items that refer to the overall organization of a knowledge system. Van Lohuizen has introduced "insight" along with understanding. We take this to be the process by which an anomaly (gap, contradiction, dissonance, an emerging new pattern) in the fabric of knowledge is detected and stimulates the generation of a question. 
Judging involves the use of values to assign priorities to units of understanding and knowledge. Deciding is essentially selecting or recognizing the instant at which to commit to a choice among viable alternatives, each of which the decider is prepared to accept.

Policymaking and planning both involve problem-solving and decision-making processes. We have analyzed problem solving into three aspects: (1) specifying a set of possible solutions, alternative objectives for policymaking, and alternative subgoals for planning; (2) specifying a set of properties or constraints a solution should satisfy. These are constraints on, or desiderata for, planned paths in the case of policymaking and optional paths between subgoals for planning; others should also be induced to do 1 and 2. (3) Specifying the knowledge necessary and sufficient to select and justify objectives and constraints for policymaking and to select a path and persuade those who are to ensure it is followed in planning.

We analyze decision making, after Simon, into three aspects. (1) Intelligence (scanning the environment and problem finding): This means identifying and diagnosing the key issues for policymakers and defining and analyzing the state-space for planners. (2) Design (invent, discover, and test solutions): This involves creating a strategy for policymakers and sketching the overall strategy and tactics to find a path in state-space (the universe of possible "states" of the system being planned for) by planners. (3) Choice (selecting and implementing a solution): This means prescribing a recommended approach by policymakers and working out a path in detail by planners.

We could ask, in each case, to what extent the proposed system is likely to (1) increase effectiveness and (2) incur expected costs. The costs include the effects of overload and diversion that are opportunity costs, the direct costs of having built, maintained, and operated the system, and the expected risks arising from failures to build the system on schedule, cost overruns, and technical shortfalls. An important step in the design of the decision support system could be an evaluation framework measuring these and related factors.

Because of the exploratory nature of this proposed system, many problems have not been clearly identified. Some major problems need at least to be analyzed in a preliminary way. One such problem is the extent to which computer conferencing (Turoff and Hiltz, 1977) enables a group to engange in a "multilogue" (a term used first by Kochen, and independently by Duke). Complex issues characterized by numerous nonquantitative random variables, interrelated probabilistically in 
irrational, unprecedented situations with high stakes (possibly with irreversible consequences) characterize such multilogues. Conferencing can probably be done, at a cost. Kochen (1981) has proposed testing new tools for inquiring conferencing communities but notes that participation parties possessing more or less issue-specific information, differing substantially in values, were probably infeasible for such an experiment. This proposal is more ambitious because it attempts to break down those barriers to linking the parties to policymaking and planning. A research agenda to help identify and test mechanisms for achieving this includes applying incentives for successful networking. The agenda would also include the design and operation of a planning-support expert system to monitor and control the potential for information overload, information distortion, or misinterpretation. Of greatest interest, perhaps, is the potential for such networks to facilitate user learning and synergistic integration of problem-solving abilities among the network participants. The explicit role of user learning in planning processes will have to be better clarified, because there are time costs (at least) incurred by learning parties. Such costs will also be distributed unequally among the participants. Ways to measure these processes against some control standard are desirable.

What are the grounds for believing that intelligence and expertise made available through a distributed computer network of the kind suggested here will improve the utilization of knowledge by planners and thus the effectiveness of planning? First, consider rationalistic planning styles in which these are appropriate: when both ends and means are fairly clearly known. To begin with, knowledge support can help planners to transform the planning situations they face into those with even more clearly known means and ends. Thereafter, the planner is provided with responses from a knowledge base and an inference engine to his or her needs for knowledge to use in specifying temporal and spatial correspondences of actions.

Second, consider incremental planning styles in situations in which both ends and means are wholly unclear. Here, no system is likely to help the planner select variables, properties that future states should have, and so on. Instead, he or she is supplied by expert systems with advice about which incremental steps to take.

One of the most significant barriers to success in the use of distributed expert systems to support planning requiring consensus among diverse stakeholders is that of incentives. It is necessary to motivate key persons to participate in the first place and to maintain communication at times 
of flagging enthusiasm and competing concerns. Attainment and maintenance of revenues, credibility, power, and influences, supporting votes are motives of various stakeholders. Well-substantiated promises to help participants achieve these goals could be adequate incentives.

Another problem concerns control over the proliferation of ideas. A large number of ideas of mediocre or bad currency may tend to drive out or drown the few ideas in good currency. Research on computer-based aids to filter out inputs of marginal value in computer conferencing or electronic mail could contribute to alleviating this problem. Peer review and other quality filters to exert some birth control on the generation of ideas may help.

Although these and similar problems confront users of such a system, on balance it is feasible, at least as a learning experiment in increasing effectiveness.

\section{Conclusion}

Our proposal to develop a planning-aid expert system has shed light on the utilization of knowledge by suggesting a role for expert systems networks. The advantages of such an approach are that it helps to bring to bear methods and concepts from information science, which is a source of critical appraisals of information system technology and potential. Problem solving in the public sector, as within business enterprises, must generate plans; competitive pressures to do so effectively are ubiquitous. The extent to which information systems and artificial intelligence will improve planning effectiveness is a topic ripe for investigation.

We make the following recommendation for realistic action: exploiting an already planned network project to realize the potential of the system we describe. European universities had an offer from IBM to set up a multipurpose network, the European Academic Research Network (EARN), connecting mainframe computers in more than 60 academic and research centers, which would also connect to BITNET in North America (Dickson, 1984). European governments trying to decide between public-sector and private-sector information network developmental pathways have delayed launching the proposed network. The value-added character of the European Academic Research Network is instructive as a prototype for widely distributed planning 
networks. We recommend investigating its use as a link between scientific research and decision making in planning. It would be best to restrict its use in such an investigation to a limited domain, probably as an experiment in the kind of mobilization of ecological knowledge called for by the recent NAS (1986) report on environmental management. An EARN-based distributed environmental management planning expert network, capitalized with knowledge-based information technologies, will, we believe, provide European environmental planners with added leverage.

Knowledge enters planning in subtle ways. Knowledge-based systems are therefore likely to be invisible and transparent: We will use tools to aid in the planning process-for example, an environmental scannerwithout awareness that computer/communication-based expert systems are hidden in the tool. The use of these systems must become so unobtrusive and intrinsic to tool use as not to divert from or interfere with performance of the task at hand. Increases in planning effectiveness are the goals and benefits of a knowledge-based distributed planning expert system. The goals and benefits are within reach of our design abilities now.

\section{References}

BENVENISTE, G. (1977) The Politics of Expertise. San Francisco: Boyd \& Fraser.

CAPLAN, N. (1984) “Research on knowledge utilization: lessons and observations." Report, CRUSK. Ann Arbor: Univ. of Michigan Press.

DICKSON, D. (1984) "Europeans look IBM gift horse in the mouth." Science 226(5 October): 4670 .

DRAKE W., R. MILLER, and D. SCHON (1983) "The study of community-level nutrition interventions: an argument for reflection-in-action." Human Systems Management 4, 2.

DUKE, R. (1984) Personal Communication.

FELDT, A. (forthcoming) "Planning theories," in J. Snyder and A. Catanese (eds.) Introduction to Urban Planning.

FISHER, R. and W. URY (1981) Getting to Yes: Negotiating Agreement Without Giving In. Boston: Houghton Mifflin.

FRIEDMANN, J. and G. ABONYI (1976) "Social learning: a model for policy research." Environment and Planning.

GORDON, M., R. BELEW, M. KOCHEN and R. LINDSAY (1986) "Information construction through computer conferencing." Presented at National Online Meeting.

HART, H. (1957) The Dark Missouri. Madison: Univ. of Wisconsin Press. 
JACOBS, H. (1984) "The knowledge household." Presented at the Third International Conference, Seattle.

KOCHEN, M. (1954) "An information-theoretical model of organizations." Trans. Inst. Radio Eng. 4: 67-75.

[ed.] (1975) Information for Action: From Knowledge to Wisdom. New York: Academic Press.

- (1980) "Opportunity costs in computer conferencing during and for economic development." Proceedings of Workshop on the Economics of Communications. Honolulu: East-West Economic Institute.

(1981) "Inquiring communities on new tools for science-based policy-making." Working paper. Berlin: Wissenschaftszentrum.

____(1982) "Barriers to planning in business." Human Systems Management 3, 4: 301-306.

- (1984) "Information about how to retrieve information: its relation to the information retrieved." Presented at second symposium on Empirical Foundations of Information and Software Sciences, Atlanta.

___ and C. BARR (1986) "Planning theory: a cognitive approach," in Interdisciplinary Planning. New Brunswick, NJ: Center for Urban Policy Research, Rutgers State University.

KOCHEN, M. and R. CRICKMAN (1978) "Citizen participation through computer conferencing." Technological Forecast. and Social Change, 1-32.

KOCHEN, M. and B. FLOYD (1983) "Degree of constraint in use of an on-line time management system." Proceedings of National Online Meeting. Medford, NJ: Learned Information.

KOCHEN, M., R. RAJAN, and Y. YUAN (1984) "Distribution of information processing for fiscal management in developmental planning." Proceedings National Online Meeting. Medford, NJ: Learned Information.

MICHAEL, D. (1973) On Learning to Plan-and Planning to Learn: The Social Psychology of Changing Toward Future-Responsive Societal Learning. San Francisco: Jossey-Bass.

National Research Council (Application of Ecological Theory to Environmental Problems Committee) (1986) Ecological Knowledge and Environmental Problem-Solving. Washington, DC: National Academy Press.

National Research Council (Geophysics Study Committee) (1982) Scientific Basis of Water Resource Management. Washington, DC: National Academy Press.

POLSBY, N. (1984) Political Innovation in America: The Politics of Policy Initiation. New Haven, CT: Yale Univ. Press.

RIDGEWAY, M. (1955) The Missouri Basin's Pick-Sloan Plan. Urbana: Univ. of Illinois Press.

SCHON, D. (1984) The Reflective Practitioner. New York: Basic Books.

TUROFF, M. and S. R. HILTZ (1977) "The process of communications and the problem of goals." Presented at 21st annual meeting, Society for General Systems Research, Denver.

VAN LOHUIZEN, W. (1984) "The knowledge household and policy making." Working Paper. The Netherlands: Eindhoven University. 
MANFRED KOCHEN was educated at MIT(1950) and at Columbia University in New York (Ph.D., applied mathematics, 1955). Since 1972 he has been Professor of Information Science, with a joint appointment as Adjunct Professor of Computer and Information Systems at the Graduate School of Business Administration, University of Michigan. His major research interests are in problems of representation of knowledge. He is the author or editor of six books, including Information for Action; he has over 150 scientific papers in technical journals or books. In 1974 he was awarded the Award of Merit by the American Society for Information Science.

CHARLES BARR(B.A., history, Yale College) is a Research Associate with the Information Science Program at the University of Michigan. His interests are in planning and policy analysis in the field of natural resources. He has worked for environmental organizations and in management science research, with an emphasis on information systems development. 